\title{
Molecular dynamics simulation of helium ion implantation into silicon and its migration
}

\author{
Lei Liu ${ }^{\mathrm{a}}$, Zongwei Xu${ }^{\mathrm{a}, *}$, Rongrong $\mathrm{Li}^{\mathrm{b}}$, Rui Zhu ${ }^{\mathrm{b}}$, Jun $\mathrm{Xu}^{\mathrm{b}, *}$, Junlei Zhao ${ }^{\mathrm{c}}$, Chao Wang ${ }^{\mathrm{d}}$, \\ Kai Nordlund ${ }^{\mathrm{c}}$, Xiu Fu ${ }^{\mathrm{a}}$, Fengzhou Fang ${ }^{\mathrm{a}}$ \\ ${ }^{a}$ State Key Laboratory of Precision Measuring Technology \& Instruments, Centre of MicroNano Manufacturing Technology, Tianjin University, Tianjin 300072, China \\ ${ }^{\mathrm{b}}$ State Key Laboratory for Mesoscopic Physics and Electron Microscopy Laboratory, School of Physics, Peking University, Beijing 100871, China \\ ${ }^{\mathrm{c}}$ Department of Physics and Helsinki Institute of Physics, University of Helsinki, P.O. Box 43, FI-00014 Helsinki, Finland \\ ${ }^{\mathrm{d}}$ State Key Laboratory of Nonlinear Mechanics, Institute of Mechanics, Chinese Academy of Sciences, Beijing 100871, China
}

\section{A R T I C L E I N F O}

\section{Keywords:}

Molecular dynamics simulation

Ion implantation

Helium

$\mathrm{Si}$

Annealing

\begin{abstract}
A B S T R A C T
In this paper, a model of helium ion implanted monocrystalline Si was constructed by using molecular dynamics (MD) simulation method to study the interaction mechanism of helium ion with monocrystalline Si and helium ion migration. In order to study the damage effect of helium ion implantation on monocrystalline Si, identify diamond structure (IDS), radial distribution function, temperature analysis were calculated and analyzed. The effects of ion doses, beam currents and energies on the damage were studied. Helium ion implanted Si with ion doses of $1 \times 10^{14} / \mathrm{cm}^{2}$ was subsequently heated to $300 \mathrm{~K}$. MD simulation results indicated that IDS damage induced by ion implantation was positively correlated with ion doses as the ion implantation increased to $1 \times 10^{14} / \mathrm{cm}^{2}$. The mean-square displacement of helium atoms was calculated during the temperature rising to $300 \mathrm{~K}$. It was found that the high permeability of helium atoms in $\mathrm{Si}$ and the acceleration of atomic thermal motion owing to elevated temperature as well as the existence of larger stress would be helpful to the migration of implant helium atoms.
\end{abstract}

\section{Introduction}

As an excellent semiconductor material, monocrystalline $\mathrm{Si}$ is increasingly used in the manufacture of semiconductor devices. However, the presence of a small amount of metal impurities in the semiconductor manufacturing process can degrade the function of the semiconductor device. It is found that the helium bubbles generated by the helium ion implantation into monocrystalline Si can form a cavity in the subsequent high temperature annealing. The inner surface of the cavity has high chemical activity due to the presence of dangling bonds which can adsorb metal impurities in the semiconductor device and improve the performance of the semiconductor device [1]. The formation of bubbles is related to the generation and evolution of defects in the process of implantation of helium ions [2]. The cavity is formed when the helium gas in helium bubbles migrates from the Si surface during high temperature annealing [3]. Therefore, it is very important to study the damage effect in the process of helium ion implantation into monocrystalline Si and the migration of helium atoms.

The bubble produced by helium ion implantation into monocrystalline Si was first discovered by Griffioen et al. [4]. In addition, a critical dose is required for the formation of bubbles. S. Godey et al. [5] conducted a helium ion implantation experiment at $1.6 \mathrm{MeV}$ and found that the threshold fluence required to form cavities was found to be between $1 \times 10^{16} \mathrm{He} / \mathrm{cm}^{2}$ and $2 \times 10^{16} \mathrm{He} / \mathrm{cm}^{2}$. Liu et al. proposed the damage evolution at different ion implantation doses: no helium bubble was formed at low ion doses even at high temperature annealing and the induced damage was He-vacancy clusters, which would be separated in the subsequent annealing; bubbles appeared at medium doses and were accompanied by high density dislocation loops; at high doses, a cavity band with a few dislocations inside it was observed [6]. Moreover, the formation of bubbles is related to ion energy, beam current and implantation temperature. Oliviero et al. studied the effect of implantation temperature and found that when ion implantation was performed at high temperature $(1073 \mathrm{~K})$, relatively low doses of helium ions could also observe the formation of bubbles in Si [7]. The growth of helium bubbles at different annealing temperatures was studied by Raineri et al. The results indicated that the diameter of the helium bubble increased with the increasing annealing temperature, and the surface peeling would occur with the increasing annealing temperature if the implantation dose was large enough [8]. Helium desorption from

\footnotetext{
* Corresponding authors.

E-mail addresses: zongweixu@tju.edu.cn (Z. Xu), xujun@pku.edu.cn (J. Xu).
} 
helium-implanted Si occurs at high temperature annealing. F. Corni et al. found that the defects affected thermal desorption were thermal unstable He-vacancy clusters, bubbles and thermally stable cavities [9]. Cerofolini et al. used the thermal desorption spectrometer (TDS) to observe the thermal desorption process of helium. It was observed that a higher temperature was attributed to the emission of helium from stable cavities while a lower temperature attributed to the emission from vacancy like defects [10].

In recent years, with the development of computer technology, molecular dynamics (MD) simulation is an effective method to study ion implantation damage [11,12]. The Ostwald Ripening (OR) and Migration\&Coarsening (MC) mechanisms have been successfully used to explain the phenomenon of bubble formation and growth caused by helium ions implantation in metallic materials [13-15]. However, due to the particularity of single crystal Si, the generation and evolution of defects and the migration of helium atoms in Si are greatly different from those in metals. Studies have shown that the defects induced by helium ions implantation into single crystal $\mathrm{Si}$ are mainly a large number of vacancy defects which are related to helium atoms. Pizzagalli et al. used the molecular dynamics (MD) simulation to simulate the formation of He-vacancy clusters. The simulation results showed that the helium implanted into the Si would form the He-vacancy complex when it met the vacancy type defect and the He-vacancy complex further captured the helium and the vacancy to form a larger He-vacancy clusters [16].

In order to explain the evolution of the defects in the monocrystalline $\mathrm{Si}$ and the migration of helium, it is very important to study the theoretical research of the Si implantation with helium ions. In this paper, a model of helium ion implanted monocrystalline Si was constructed by using molecular dynamics (MD) simulation to study the interaction mechanism of helium ion with monocrystalline Si and the damage mechanism as well as helium ion migration.

\section{MD simulation detail and methods}

\subsection{MD simulation detail}

Large-scale Atomic/Molecular Massively Parallel Simulator (LAMMPS) code was used to simulate ion implantation. A Tersoff type potential [17] is used to describe the interactions between Si-Si. It can provide good equilibrium properties and has been proven accuracy and simulate the interactions between $\mathrm{Si}-\mathrm{Si}$. The interaction potential between He-He adopted in this study was a Table type potential developed by Juslin et al. [18], and the $1 \mathrm{j} /$ cut type potential is used to describe the interactions between $\mathrm{He}-\mathrm{Si}$. The $\mathrm{lj} / \mathrm{cut}$ styles compute the standard 12/6 Lennard-Jones potential. The parameter $r$ is the distance between atoms, and $\varepsilon$ and $\sigma$ are potential energy parameters:

$\mathrm{E}=4 \varepsilon\left[\left(\frac{\sigma}{\mathrm{r}}\right)^{12}-\left(\frac{\sigma}{\mathrm{r}}\right)^{6}\right]$

where $\varepsilon=1 \mathrm{eV}, \sigma=1$ Angstrom, $\mathrm{r}=2.5$ Angstrom.

Fig. 1 showed the molecular dynamics (MD) model for ion implantation with the dimensions of $22 \mathrm{a}_{0} \times 32 \mathrm{a}_{0} \times 32 \mathrm{a}_{0}$, including 182,272 atoms. The model consists of a boundary fixed layer, a thermo layer and a newton layer. The boundary layer was used to fix the whole bulk, the thermo layer was used to maintain the temperature of the model and the newton layer satisfied the second newton's law. The periodic boundary conditions were used in y-direction and z-direction while fixed boundary conditions were applied in the x-direction. Considering the simulation speed and simulation accuracy, the simulation method with variable time step was used in the simulation $[19,20]$. The timestep was increased from $0.001 \mathrm{fs}$ to $1 \mathrm{fs}$ during the simulation to ensure that the largest movement distance of all particles in the system were less than $0.02 \AA$ during the single integration process $[20,21]$.

100 Helium ions were implanted into single crystal Si consecutively

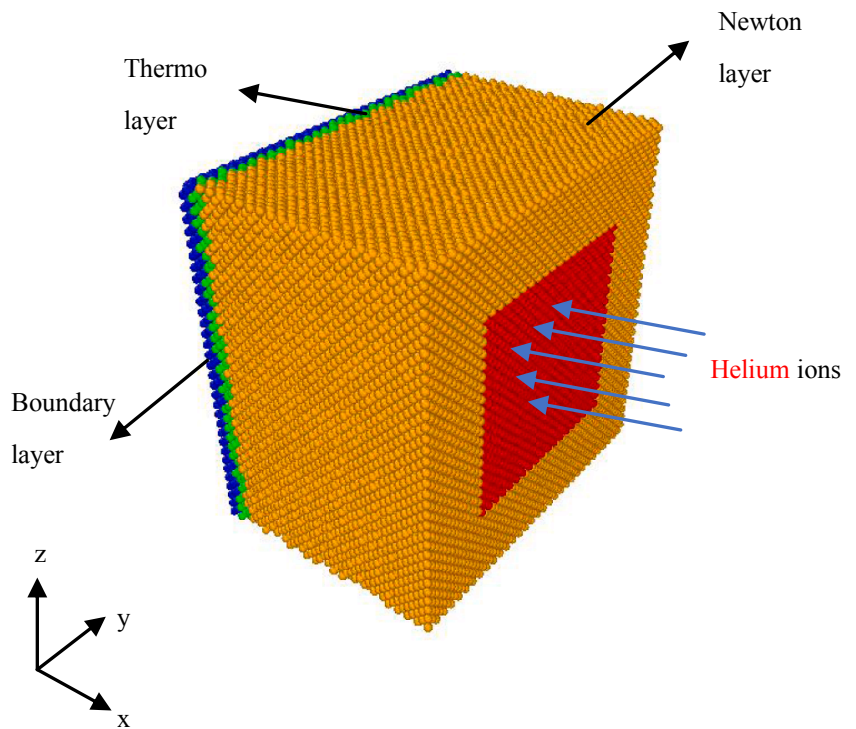

Fig. 1. The MD model for ion implantation with the dimensions of $22 \mathrm{a}_{0} \times 32 \mathrm{a}_{0} \times 32 \mathrm{a}_{0}$, including 182,272 atoms. 100 helium ions with $500 \mathrm{eV}$ were implanted into the red square region with the size of $10 \mathrm{~nm} \times 10 \mathrm{~nm}$. The ion implantation rate is $1557 \AA / \mathrm{ps}$, and the implantation dose is $1.0 \times 10^{14}$ ions $/ \mathrm{cm}^{2}$. (For interpretation of the references to colour in this figure legend, the reader is referred to the web version of this article.)

(one by one). One ion was implanted each time and another after the temperature relaxing down to the set temperature. The emission time interval between two adjacent ions is $60 \mathrm{ps}$. The ion implantation rate is $1557 \AA / \mathrm{ps}$, and the implantation dose is $1.0 \times 10^{14}$ ions $/ \mathrm{cm}^{2}$. After 100 helium ions with $500 \mathrm{eV}$ were implanted into single crystal $\mathrm{Si}$, the temperature of the model was heated to $300 \mathrm{~K}$. The periodic boundary conditions were used in $\mathrm{x}$-direction, $\mathrm{y}$-direction and $\mathrm{z}$-direction with a Nose-Hoover thermostat [22,23] (NPT ensemble with 0 bar pressure). The system timestep was set to $0.5 \mathrm{fs}$ due to the acceleration of atomic thermal motion at the high temperature. Detailed ion implantation parameters and subsequent heating parameters were shown in Table 1.

\subsection{Analysis parameters}

Stress is a basic parameter that adopted to characterize material behavior during ion implantation. Stress analysis $[24,25]$ is based on formula (2).

$\sigma_{\alpha \beta} i=\sum \frac{m_{i} v_{i}^{\alpha} v_{i}^{\beta}}{V_{i}}+\frac{1}{2 V_{i}} \sum f_{i j}^{\alpha} r_{i j}^{\beta}$

where $m_{i}$ represents the mass of the $i^{\text {th }}$ atom; $V_{i}$ is the volume of space in which the $\mathrm{i}^{\text {th }}$ atom is located; $\mathrm{v}_{i}^{\alpha}, \mathrm{v}_{i}^{\beta}$ represents the velocity component of $i^{\text {th }}$ atom in the $\alpha$-direction and $\beta$-direction, respectively; $f_{i j}^{\alpha}$ represents the component of the force on the $\mathrm{i}^{\text {th }}$ atom exerted by the $\mathrm{j}^{\text {th }}$ atom in the $\alpha$ direction; $r_{i j}^{\beta}$ represents the distance between the $\mathrm{i}^{\text {th }}$ atom

Table 1

Simulation parameters for ion implantation and heating.

\begin{tabular}{ll}
\hline Condition & Parameters \\
\hline Work material & $\mathrm{Si}(100)$ \\
Lattice constant & $\mathrm{a}_{0}=5.431 \AA$ \\
Dimensions & $22 \mathrm{a}_{0} \times 32 \mathrm{a}_{0} \times 32 \mathrm{a}_{0}$ \\
Bulk temperature & $0 \mathrm{~K}$ \\
Number of He ions & 100 \\
Incident angle & $7^{\circ}$ \\
Implant area & $10 \mathrm{~nm} \times 10 \mathrm{~nm}$ \\
Heating temperature & $300 \mathrm{~K}$ \\
Thermostat style & Nose-Hoover thermostat (NPT ensemble)
\end{tabular}




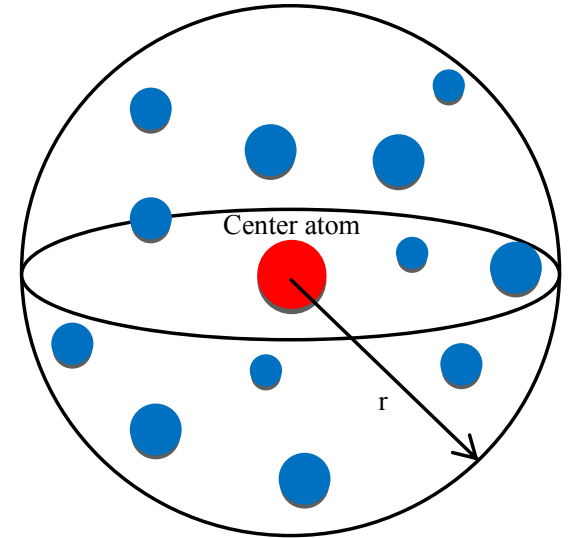

Fig. 2. Schematic diagram of the calculation of local temperature and stress.

and the $\mathrm{j}^{\text {th }}$ atom in the $\beta$ direction. In the MD simulation, since it is difficult to calculate the volume of a single atom, the stress of a single atom is approximated based on the formula (2). As shown in Fig. 2, the red particle is the center of the sphere with a volume $\mathrm{V}$. The sum of all atomic stress tensors in the sphere is given to the center atom. After calculating the stress in each direction of the single crystal $\mathrm{Si}$, the hydrostatic pressure was obtained by the formula (3)[26], so as to analyze the overall stress state of the material. The calculation of temperature is based on formula (4).

$\sigma_{\text {hydro }}=\left(\sigma_{x x}+\sigma_{y y}+\sigma_{z z}\right) / 3$

$E_{K}=\frac{3}{2} N K_{B} T$

where $E_{\mathrm{k}}$ is kinetic energy, $N$ is the number of atoms in the sphere, $K_{\mathrm{B}}$ is the Boltzmann constant, and $T$ is the temperature. The temperature calculation is the same as the stress calculation method. The meansquare displacement is used to illustrate the migration characteristics of atoms which is defined as follows:

$m s d=\frac{1}{N} \sum_{i=1}^{N}\left(d_{x i}^{2}+d_{y i}^{2}+d_{z i}^{2}\right)$

where $d_{\mathrm{xi}}, d_{\mathrm{yi}}, d_{\mathrm{zi}}$ represents the displacement of the $\mathrm{i}^{\text {th }}$ atom in the $\mathrm{x}$ - direction, y-direction and z-direction, respectively. $N$ is the number of atoms. The Identify Diamond Structure (IDS) method [27] is used to identify atoms that are arranged in a cubic or hexagonal diamond lattice. The IDS damage in this paper includes atoms that are arranged in a hexagonal diamond and unknown lattice. In addition, the coordination number and radial distribution function were also used to study the lattice structure of the material. The results of MD simulations were visualized and analyzed by using OVITO [28] which is a scientific visualization and analysis software for atomistic and particle simulation data.

\section{Results and discussion}

\subsection{Helium ion implantation into Si}

The temperature, potential energy and IDS damage during the single ion implantation into Si are shown in Fig. 3. When the helium ion began to enter the monocrystalline $\mathrm{Si}$, the cascade collisions occurred, which caused the temperature and potential energy of the newton layer to rise rapidly. Then the IDS damage increased sharply, and the potential energy also reached the highest point when the damage reached the maximum. After the IDS damage reached the highest point, there would be a stage of sharp decrease in damage, it was considered that if the incident energy in the cascade collision was higher than the threshold displacement energy of the atom, the atom would be knocked out of the lattice site to form interstitial atom and produced a vacancy on the lattice site [29]. Fig. 4 shows temperature distribution during ion implantation into single crystal Si. At $4.2 \mathrm{ps}$, the maximum damage with maximum local temperature of $600 \mathrm{~K}$ was reached. There was no significant difference in IDS damage between $15 \mathrm{ps}$ and $80 \mathrm{ps}$. This also met the IDS damage curve, but the temperature continued to decrease under a Berendsen thermostat.

Fig. 5 showed the damage evolution of monocrystalline Si under different ion doses. As the ion doses increased, the scope of the amorphous region of the Si substrate had also extended. The calculation results of the radial distribution function (RDF) were shown in Fig. 6. In order to verify the amorphization of the irradiation region, the selected calculation region size is $5 \mathrm{~nm} \times 5 \mathrm{~nm} \times 5 \mathrm{~nm}$, as shown in the red square region in Fig. 5(d). After ion implantation with the dose of $10 \times 10^{12} / \mathrm{cm}^{2}$ (10 impacts), the RDF curve did not change significantly because the proportion of IDS damage atoms in the selected

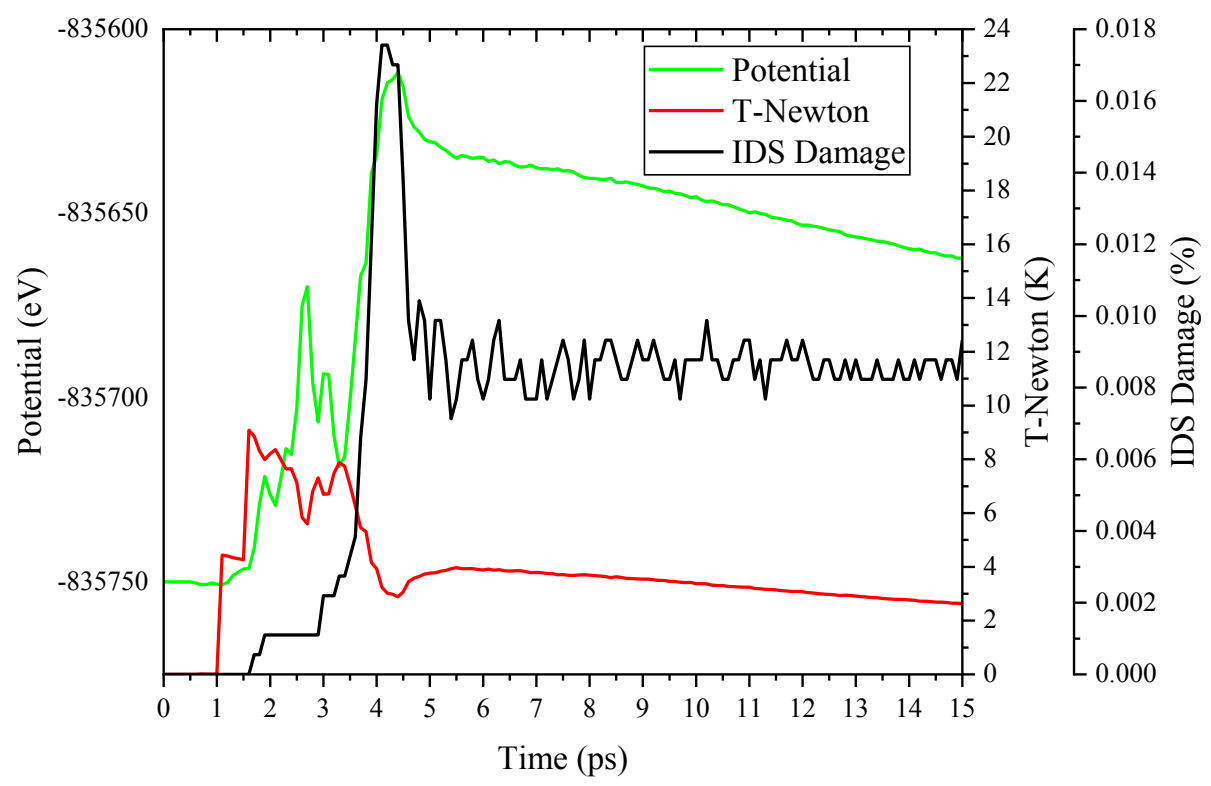

Fig. 3. The temperature, potential energy and IDS damage during the single ion implantation into silicon. The graph only shows the evolution of the first 15 ps during ion implantation into single crystal silicon. 


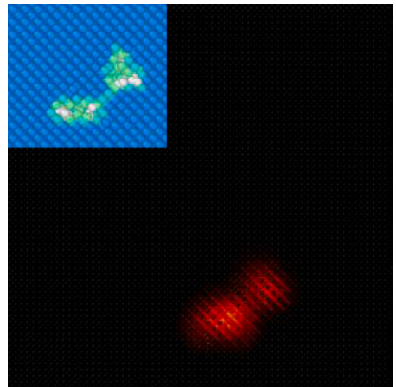

a) $4.2 \mathrm{ps}$

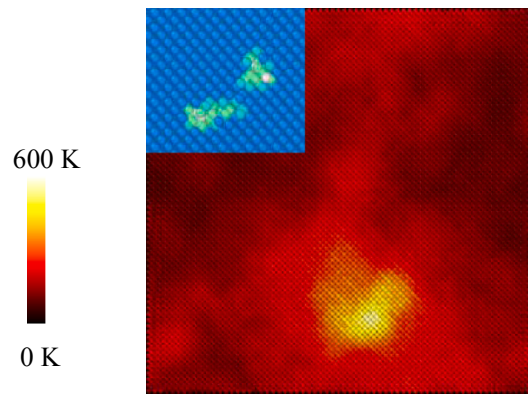

b) $15 \mathrm{ps}$

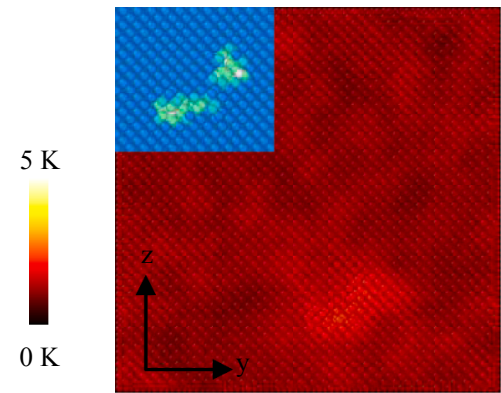

$5 \mathrm{~K}$

c) $80 \mathrm{ps}$

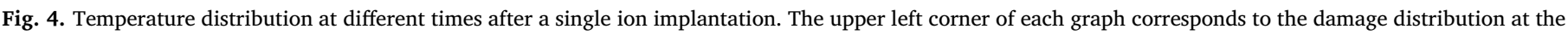
same time.

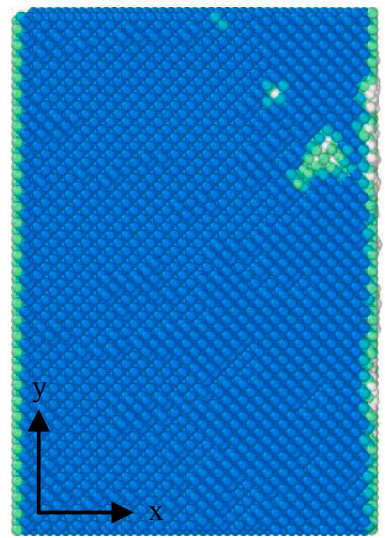

a)10 impacts

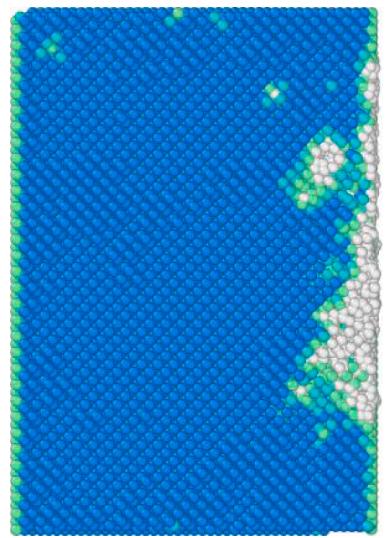

b) 40 impacts

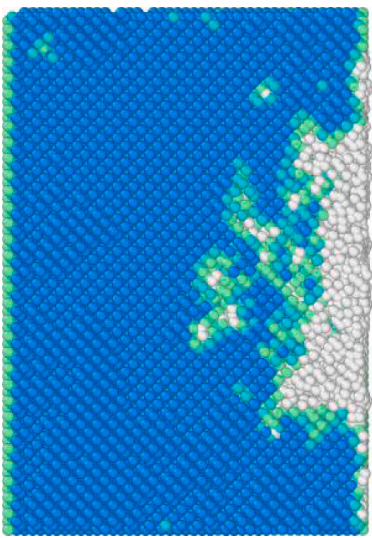

c) 80 impacts

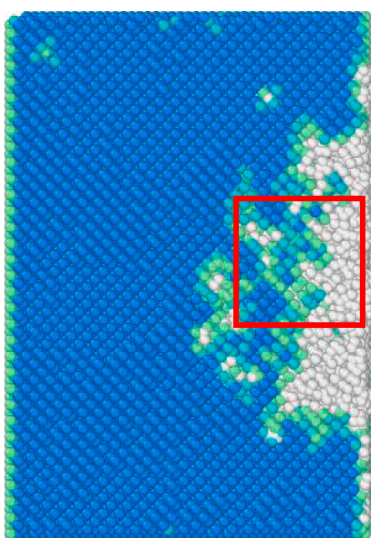

d) 100 impacts

Fig. 5. Damage distribution at different ion doses. The white atom represents the damaged atom.

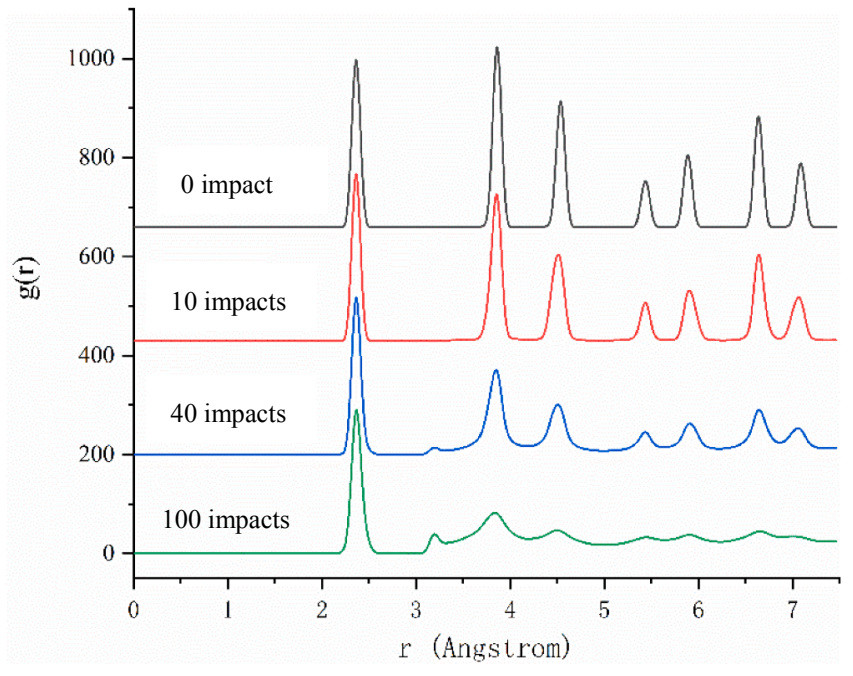

Fig. 6. Radial distribution function at different ion doses. For 0 impact, 10 impacts and 40 impacts, the curves are shifted upwards.

region was very low $(0.85 \%)$. When the ion implantation dose increased to $40 \times 10^{12} / \mathrm{cm}^{2}$ (40 impacts), the proportion of IDS damage atoms was $16.7 \%$ at this time, some Si atoms drifted and the periodic distribution was destroyed after the collision between the helium ion and the $\mathrm{Si}$ atom in the equilibrium position. It was characterized by a weaker peak height and an increased peak width of the RDF. After ion implantation with the dose of $100 \times 10^{12} / \mathrm{cm}^{2}$ (100 impacts), the proportion of IDS damage atoms was $49.3 \%$ at this time. The regularity of periodic distribution was severely damaged, and the amorphous characteristics of short-range ordered and long-range disordered were reflected.

The IDS damage and the longitudinal depth distribution of the damaged atom in the $\mathrm{x}$-direction at different beam currents are shown in Fig. 7(a) and (b), respectively. It was found that the IDS damages had a slight change when the beam current increased from $2.67 \mathrm{nA}$ to $5.33 \mathrm{nA}$. However, the longitudinal damage depth of the damaged region increased with increasing the beam current. This result showed the same trend with the experimental results [30], which showed that larger dwell time would produce more damages under the same ion dose. Larger beam dwell time and current would be helpful to depress the ion implantation induced damages' self-annealing, and produce more damages.

The IDS damage and the longitudinal depth distribution of the damaged atom in the $\mathrm{x}$-direction at different energies are shown in Fig. 8(a) and (b). The IDS increased significantly as the beam energy increased from $500 \mathrm{eV}$ to $1000 \mathrm{eV}$, and the IDS gap became more and more obvious as the ion doses increased. The evolution of surface topology with different energy and beam current were shown in Fig. 9. Ions bombarded the sample surface created pits at the point of incidence and swelling surrounding the pits. The change in the number of swelling and pits was not very obvious when the beam current increased from $2.67 \mathrm{nA}$ to $5.33 \mathrm{nA}$ as shown in Fig. 9 (a) and (b), while the swelling increased distinctly as the beam energy increased from $500 \mathrm{eV}$ to $1000 \mathrm{eV}$ exhibited in Fig. 9(a) and (c). Helium ion implantation experiment was performed on n-type Si wafer at $30 \mathrm{KeV}$ beam energy. TEM microstructural features of the ion implanted sample clearly showed the surface swelling results above the Helium ion implantation area, as shown in Fig. 9(d). 


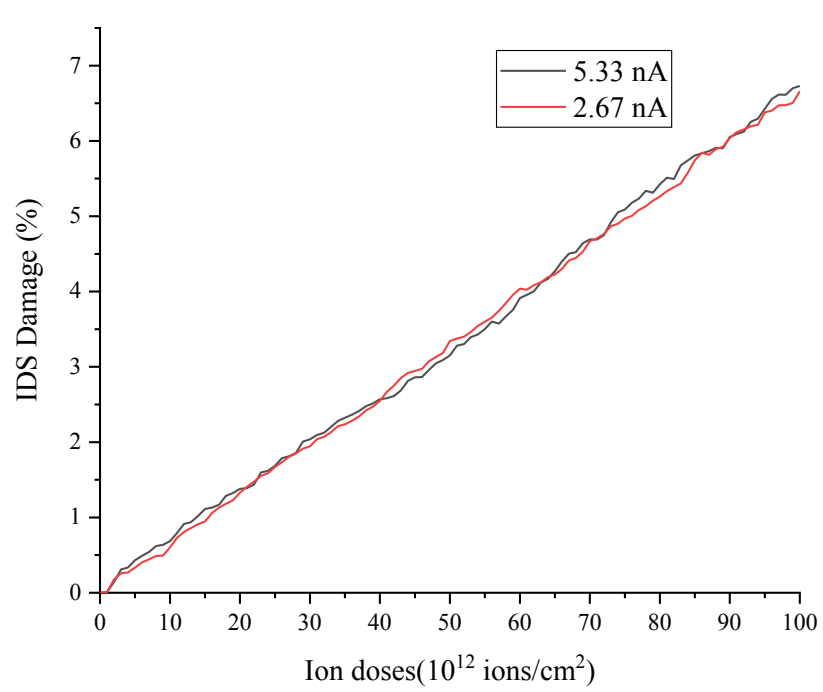

a) IDS evolution at different beam currents.

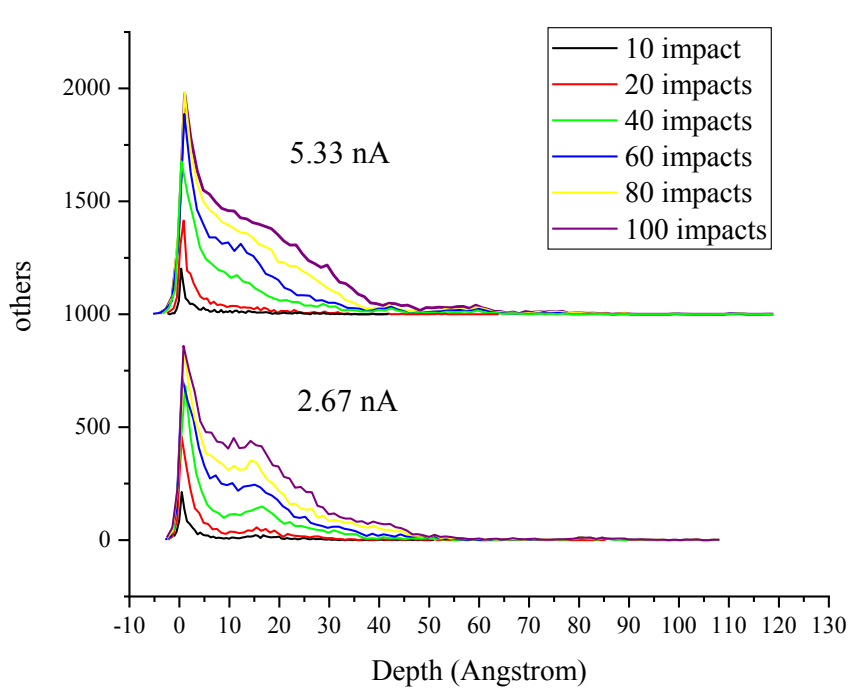

b) Longitudinal distribution of damaged atoms in the $\mathrm{x}$-direction.

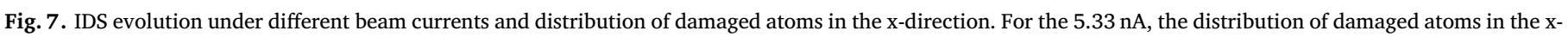
direction is shifted upwards.

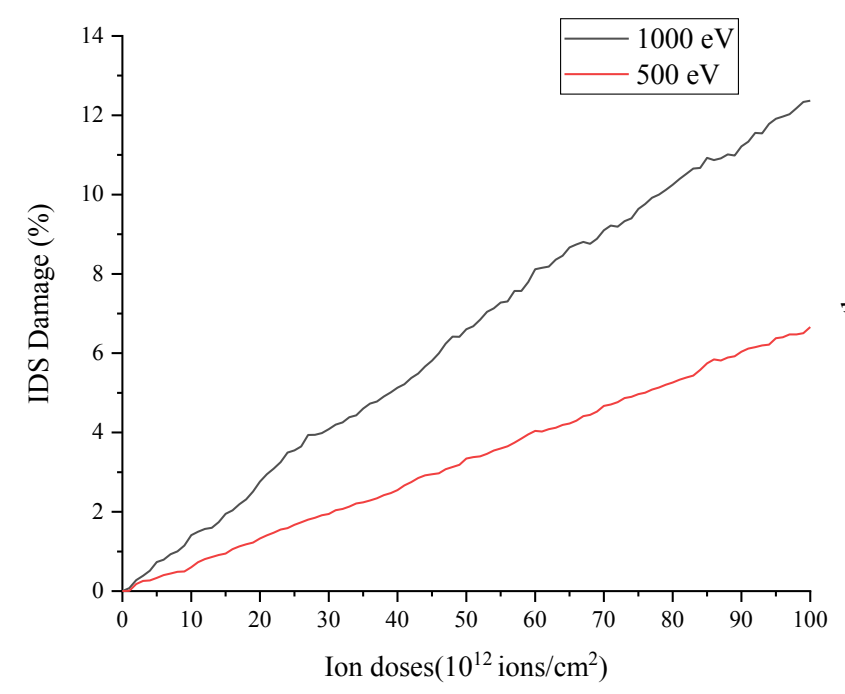

a) IDS evolution at different ion energy

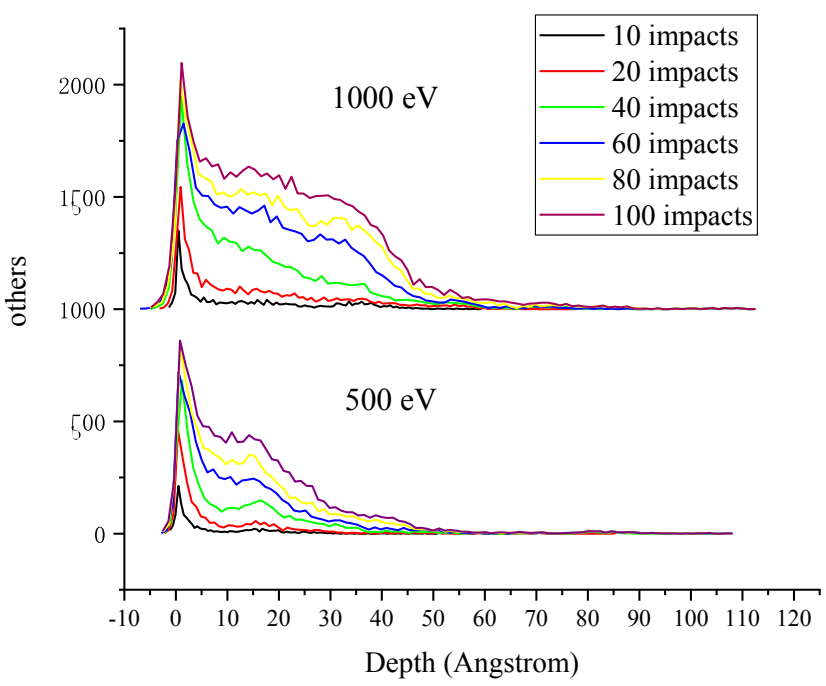

b) Longitudinal distribution of damaged atoms in the $\mathrm{x}$-direction.

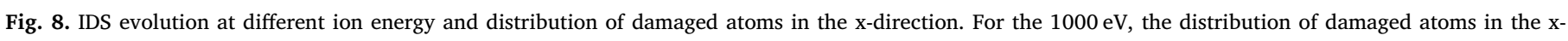
direction is shifted upwards.

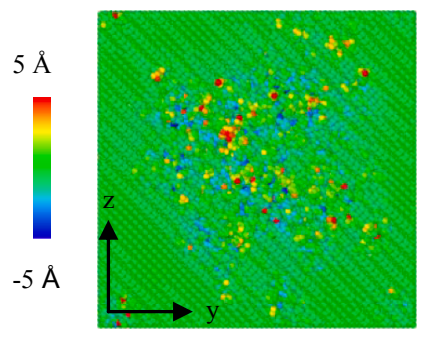

a) $2.67 \mathrm{nA}, 500 \mathrm{eV}, 100$ impacts

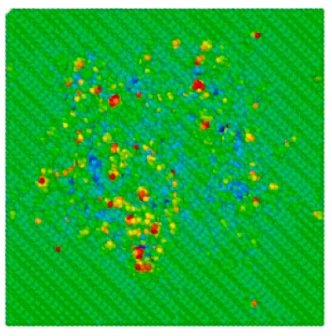

b) $5.33 \mathrm{nA}, 500 \mathrm{eV}, 100 \mathrm{impacts}$

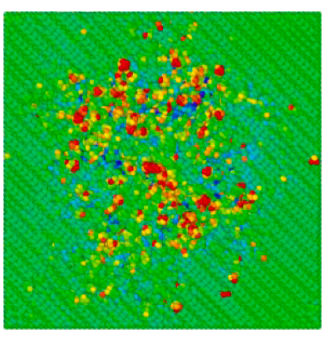

c) $2.67 \mathrm{nA}, 1000 \mathrm{eV}, 100$ impacts

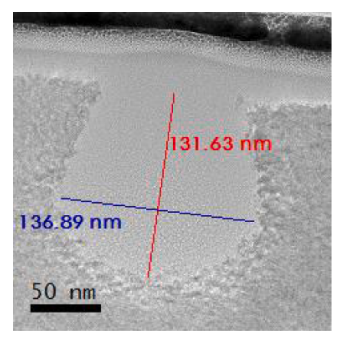

d) Swelling afer Helium implants

Fig. 9. Evolution of surface topology during ion implantation with different beam current and different energy.

\subsection{Migration of helium ions after implantation}

After 100 helium ions with $500 \mathrm{eV}$ implanted into single crystal Si, the temperature of the model gradually increased to $300 \mathrm{~K}$. The process is divided into five periods: $0-50 \mathrm{ps}$ is $0 \mathrm{~K}$ relaxation stage, $50-200 \mathrm{ps}$ is temperature-rising stage, where temperature is increased to $300 \mathrm{~K}$, the temperature of $200-1700 \mathrm{ps}$ is maintained at $300 \mathrm{~K}$. The curves of potential energy, temperature, IDS damage and mean-square displacement of helium and $\mathrm{Si}$ atoms as function of time were shown in Fig. 10. The damage aggravated with the increase of temperature and the 


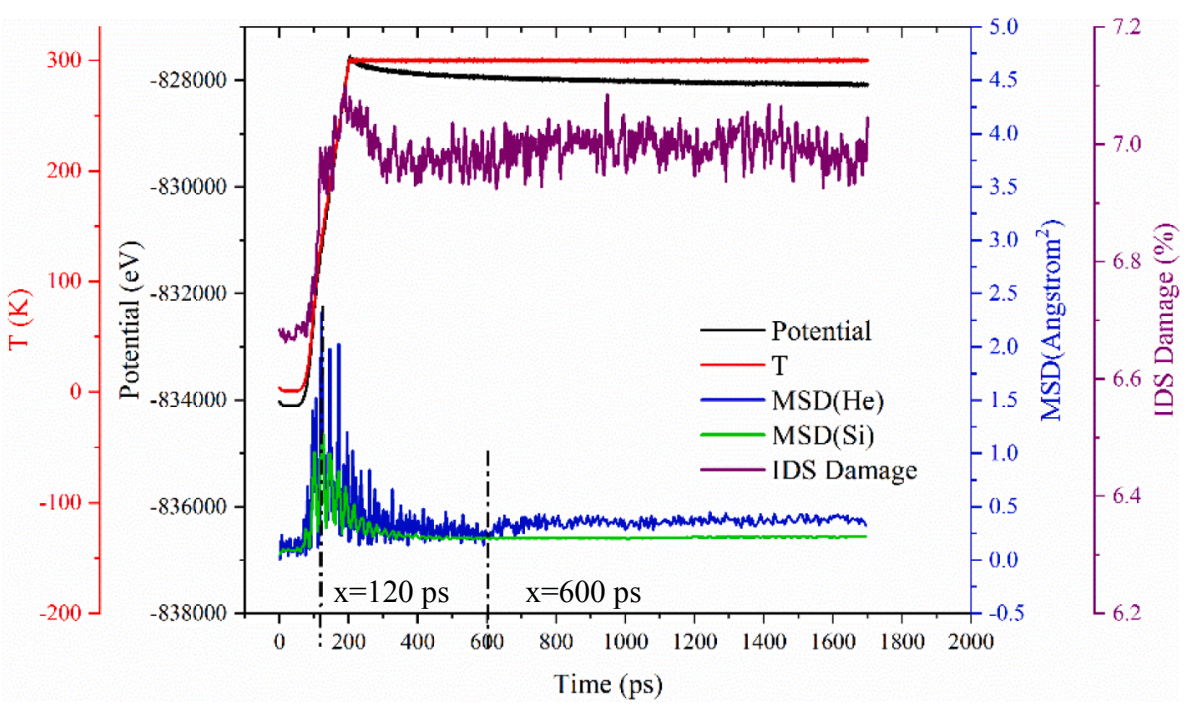

Fig. 10. The evolutions of potential energy, temperature, IDS damage and mean-square displacement of helium and silicon atoms.

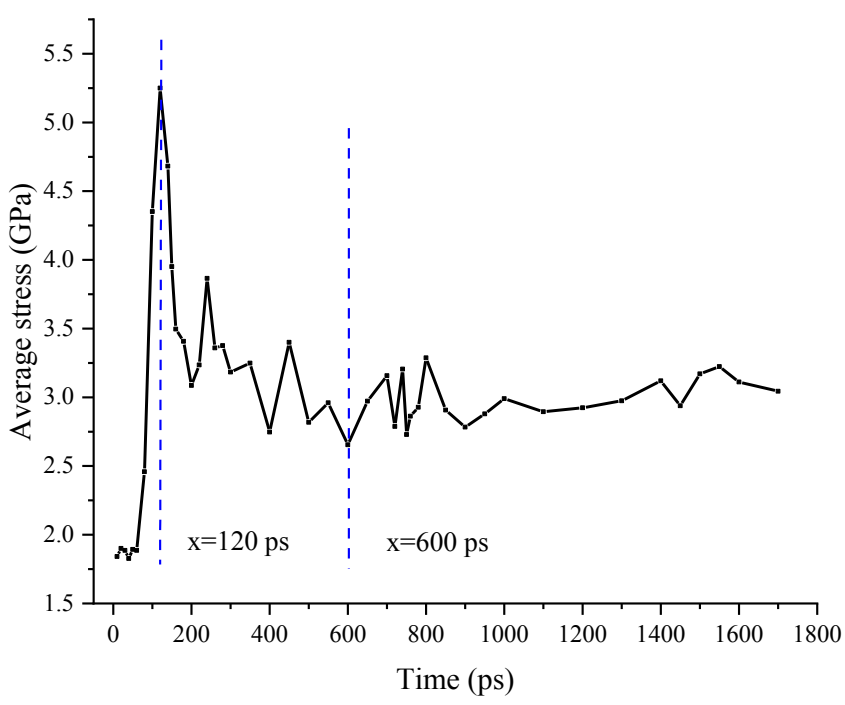

Fig. 11. The variation of average stress value of 100 implanted helium atoms with time.

potential energy also reached the highest point when the damage reached the maximum at $200 \mathrm{ps}$. After the IDS damage reached the maximum value, a stage of sharp decrease in damage was appeared due to the recrystallization of some amorphous Si atoms. Finally, IDS damage fluctuated at a certain value. The mean-square displacement (MSD) is used to illustrate the motion characteristics of helium atoms and $\mathrm{Si}$ atoms. The MSD of helium atoms increased first and then decreased with the increasing temperature, and the MSD of helium atoms had a larger fluctuation in the temperature-rising stage. Finally, the MSD curve fluctuated at a fixed value with a small amplitude in the $300 \mathrm{~K}$ temperature-holding stage. Comparing with the beginning, it could be observed that the MSD of the helium atoms at the stable time increased which indicated that the helium atoms implanted into the single crystal Si migrated at a temperature of $300 \mathrm{~K}$. In addition, we also found there was an increase of the MSD of Si atoms which was smaller than the helium atoms in contrast to the beginning.

In order to study the effect of local stress on the migration of helium atoms, we analyzed the stress state of the helium atoms during the heating process. The average stress versus time curve for helium atoms is shown in Fig. $11.0-50 \mathrm{ps}$ was the $0 \mathrm{~K}$ relaxation stage and the average stress remained basically unchanged. The average stress rose sharply from $50 \mathrm{ps}$ to $120 \mathrm{ps}$. It was found that the MSD of helium atoms increased at this stage. The average stress was in a downward trend as the same as the MSD curve of helium atoms from 120 ps to 200 ps. After $400 \mathrm{ps}$, the average stress of helium atoms was nearly the same around $3.0 \mathrm{Gpa}$ and the MSD of helium atoms increased slightly at $600 \mathrm{ps}$ and remained stable after then. What's more, it had been found that helium atoms had high permeability in $\mathrm{Si}[16,31]$. Therefore, it could be considered that the migration of helium atoms in monocrystalline $\mathrm{Si}$ was the combination results of the high permeability of helium atoms in $\mathrm{Si}$, the acceleration of atomic thermal motion at higher temperatures and the stress-induced movement of helium atoms.

\section{Conclusions}

In this paper, molecular dynamics simulation was used to study the helium ion implanted single crystal Si and the migration. Identify diamond structure, radial distribution function, temperature analysis and atomic stress analysis were calculated and analyzed. The effects of different beam current and energy are also considered. Our work has a further understanding of helium ion implanted into monocrystalline Si. The main results are summarized as follows: firstly, the longitudinal damage depth of the damaged region increased when the beam current increased from $2.67 \mathrm{nA}$ to $5.33 \mathrm{nA}$. Secondly, the surface topology of the monocrystalline $\mathrm{Si}$ can be changed by the implantation of helium ions. Molecular dynamics simulation and helium ion implantation experiment all showed that helium ions bombarding the sample surface created pits at the point of incidence and swelling surrounding the pits. Thirdly, the high permeability of helium atoms in Si and the acceleration of atomic thermal motion owing to elevated temperature as well as the existence of larger stress would be helpful to the migration of implant helium atoms.

\section{Acknowledgements}

The study was supported by the National Natural Science Foundation of China (No. 51575389, 51511130074, 11327902, 11605001), National Natural Science Foundation of China (NSFC) German Research Foundation (DFG) International Joint Research Programme (51761135106), the Natural Science Foundation of Tianjin (15JCYBJC19400), State key laboratory of precision measuring technology and instruments (Pilt1705), and the ' 111 ' project by the State Administration of Foreign Experts Affairs and the Ministry of Education of China (Grant No. B07014). 


\section{References}

[1] V. Raineri, M. Saggio, E. Rimini, Voids in Si by He implantation: from basic to applications, J. Mater. Res. 15 (7) (2000) 1449-1477.

[2] R.S. Brusa, G.P. Karwasz, N. Tiengo, et al., He-implantation induced defects in Si studied by slow positron annihilation spectroscopy, J. Appl. Phys. 85 (4) (1999) 2390-2397.

[3] J. Grisolia, A. Claverie, G.B. Assayag, et al., Growth mechanism of cavities in $\mathrm{MeV}$ helium implanted Si, J. Appl. Phys. 91 (11) (2002) 9027-9030.

[4] C.C. Griffioen, J.H. Evans, P.C. de Jong, et al., Helium desorption/permeation from bubbles ins ilicon: a novel method of void production, Nucl. Instr. And Meth. B 27 (1987) 417-420.

[5] S. Godey, T. Sauvage, E. Ntsoenzok, et al., Cavities and dislocations induced in Si by Me V He implantation, J. Appl. Phys. 87 (2000) 2158-2161.

[6] C.L. Liu, R. Delamare, E. Ntsoenzok, et al., Evolution of nucleation sites and bubble precursors in Si as a function of helium implanted dose, Mat. Res. Soc. Symp. Proc. 719 (2002) 229-234.

[7] E. Oliviero, Marie-Laure David, Marie Beaufort, J.F. Barbot, A. van Veen, On the effects of implantation temperature in helium implanted Si, Appl. Phys. Lett. 81 (2002) 4201-4203.

[8] V. Raineri, P.G. Falica, G. Percolla, et al., Gettering of Metals by Voids in Si, J. Appl. Phys 78 (1995) 3725-3735.

[9] F. Corni, G. Calzolari, F. Gambetta, et al., Evolution of vacancy-like defects in helium-implanted (100) Si studied by thermal desorption spectrometry, J. Mater. Sci. Eng. B 71 (1) (2000) 207-212.

[10] G.F. Cerofolini, G. Calzolari, F. Corni, et al., Thermal desorption spectra from cavities in helium-implanted Si, Phys. Rev. B 61 (2000) 10183-10193.

[11] B.L. Zhang, J. Wang, M. Li, et al., A molecular dynamics study of helium bubble formation and gas release near titanium surfaces, J. Nucl. Mater. 438 (1-3) (2013) $178-182$.

[12] Heng-Feng Gong, Min Liu, Fei Gao, et al., Helium nano-bubble bursting near the nickel surface, J. Chin. Phys. B 11 (2017) 155-161.

[13] H. Schroeder, P.F.P. Fichtner, On the coarsening mechanisms of helium bubbles ostwald ripening versus migration and coalescence, J Nucl. Mater. 179-181 (3) (1991, s) 1007-1010.

[14] S. Frabboni, F. Corni, C. Nobili, et al., Nanovoid formation in helium-implanted single-crystal Si studied by in situ techniques, J. Phys. Rev. B 69 (16) (2004) 1124-1133.
[15] J.H. Evans, Mechanisms of void coarsening in helium implanted Si, J. Nuc. Inst. Methods Phys. Res. B 196 (1) (2002) 125-134.

[16] L. Pizzagalli, M.L. David, M. Bertolus, Molecular dynamics simulation of the initial stages of He bubbles formation in Si, J. Modelling Simul. Mater. Sci. Eng. 21 (6) (2013) 065002.

[17] J. Tersoff, New empirical approach for the structure and energy of covalent systems, J. Phys. Rev. B Condens. Matter 37 (12) (1988) 6991-7000.

[18] N. Juslin, B.D. Wirth, Interatomic potentials for simulation of He bubble formation in W, J. Nucl. Mater. 432 (1-3) (2013) 61-66.

[19] K. Nordlund, A. Kuronen, Molecular dynamics simulation of ion ranges at keV energies, J. Nucl. Instrum. Methods Phys. Res. 115 (1-4) (1996) 528-531.

[20] Y.J. Xiao, F.Z. Fang, Z.W. Xu, X.T. Hu, Annealing recovery of nanoscale Si surface damage caused by Ga focused ion beam, Appl. Surf. Sci 343 (2015) 56-69.

[21] X. Fu, Z. Xu, Z. He, et al., Molecular dynamics simulation of silicon ion implantation into diamond and subsequent annealing, Nucl. Instrum. Methods Phys. Res. (2018).

[22] S. Nose, A unified formulation of the constant temperature molecular dynamics methods, J. Chem. Phys. 81 (1) (1984) 511-519.

[23] W.G. Hoover, Canonical dynamics: equilibrium phase-space distributions, J. Phys. Rev. A Gen. Phys. 31 (3) (1985) 1695-1697.

[24] B. Liu, X. Qiu, How to compute the atomic stress objectively, J. Comput. Theoretical Nanosci. 6 (5) (2008) 1081-1089(9).

[25] BinLiu RanXu, Investigation on applicability of various stress definitions in atomistic simulation, J. Acta Mech. Solida Sin. 22 (6) (2009) 644-649.

[26] F. Wakai, Y. Shinoda, T. Akatsu, Tensor-virial equation for deformation of a particle in viscous sintering, J. Am. Ceramic Soc. 95 (9) (2012) 2785-2787.

[27] E. Maras, O. Trushin, A. Stukowski, et al., Global transition path search for dislocation formation in Ge on Si (001), J. Comput. Phys. Commun. 205 (2016) 13-21.

[28] A. Stukowski, Visualization and analysis of atomistic simulation data with OVITOthe Open Visualization Tool, J. Modelling Simul. Mater. Sci. Eng. 18 (6) (2010) 2154-2162.

[29] G.S. Was, Fundamentals of radiation materials science, Fundamentals of Radiation Materials Science, Springer, 2007.

[30] Z.W. Xu, F.Z. Fang, Y.Q. Fu, et al., Fabrication of micro/nano-structures using focused ion beam implantation and XeF2 gas-assisted etching, J. Micromech. Microeng. 19 (5) (2009) 054003.

[31] G.F. Cerofolini, F. Corni, S. Frabboni, et al., Hydrogen and helium bubbles in Si, J. Mater. Sci. Eng. R Rep. 27 (1-2) (2000) 1-52. 